\title{
Digital Learning Tools for Mobile Devices for Accomplish Hypothesis Testing of Statistical Parameters
}

\author{
https://doi.org/10.3991/ijim.v13i06.10436 \\ Francisco Javier Tapia-Moreno $(\varpi)$ \\ Universidad de Sonora, Hermosillo, Mexico \\ ftapia@mat.uson.mx \\ Hector Antonio Villa-Martinez \\ Universidad de Sonora, Hermosillo, Mexico
}

\begin{abstract}
Technological changes have been associated with the evolution of computer and telecommunications systems. These changes have resulted in a rethinking of teaching and learning methods in the new digitalized environment at all educational levels. This rethinking motivates some teachers to design new digital tools that support students in their learning process, offering them an easier and more entertaining way to obtain knowledge. The digital learning tools are software and informatics programs that make everyday activities easier for students. We have designed four digital learning tools for the learning of inferential statistics that allow college students to perform hypothesis tests for: $i$ ) the arithmetic mean of the population, ii) the proportion of a population, iii) the difference between two arithmetic means, and $i v$ ) the difference between two proportions. These digital learning tools are products from the project "Statisticsto-Go" that is being developed at the University of Sonora. This project is now in its fourth stage.
\end{abstract}

Keywords-Digital learning tools, u-learning, m-learning, hypothesis test, statistics.

\section{Introduction}

In the Institutional Development Plan 2017-2021 and the Educational Model 2030 of the University of Sonora, it is mentioned that the current context presents the university with the opportunity to strengthen educational programs of a technological nature, and link them to the productive sector through cooperative schemes and under new modalities and teaching options, which use the new information and communication technologies in open and distance education modalities $[1,2]$.

At the University of Sonora, several bachelor's degrees have inferential statistics courses that include the subject of hypothesis testing. In statistics, a hypothesis is a statement about a population parameter such as the arithmetic mean, the standard deviation, the proportion, or the correlation coefficient. A hypothesis test is a standard procedure to test an assertion about one of the aforementioned parameters. In this test 
there are two possible outcomes: the first is to reject our hypothesis and the second is not to reject this hypothesis because there is statistical evidence not to do so.

Currently, a form of learning is mobile learning (or m-learning) that facilitates the construction of knowledge, problem solving, and the development of skills in an autonomous and ubiquitous way, thanks to the use of portable devices, like laptops, smartphones, tablets, and in general any device that has some form of wireless connectivity. m-learning is e-learning (education through Internet) but in mobile devices. In a similar way, u-learning (ubiquitous learning) is any scenario in which the students can get totally immersed in the learning process. Therefore, a learning ubiquitous environment is a situation or a generalized or omnipresent context where students can be learning without being completely conscious of the process [3].

Digital Learning Tools (DLTs) are software that make easy our quotidian life. DLTs are useful for complementing the process of teaching and learning [4]. They support the teacher either in the classroom or out of it because students can obtain the required knowledge at their own pace and in their own time. These tools also propitiate autonomous learning, promotes auto study with the help of the communications technology.

The above mentioned and the success obtained in the three previous stages, has motivated us to add a fourth stage to the project "Statistics-to-Go". In the previous stages we designed DLTs for mobile devices using Android operating systems, in order to generate diagrams like bar charts, histograms, pie charts, frequency polygons, and scatterplots; compute measures such as range, the arithmetic mean, median, mode, percentiles, standard deviation, coefficient of variation, coefficients of asymmetry and of curtosis of Fisher; compute confidence intervals for the arithmetic mean of population, proportion of a population, the difference among two arithmetic means, the difference among two proportions, and the variance and the standard deviation of the population $[5,6]$.

In this fourth stage, we designed four DLTs for inferential statistics. With these tools, college students can perform tests of hypothesis. These objects are very easy to use, students just enter the required statistical data in the DLT, choose one of the four options for $H_{0}$, and press "Compute" and interpret the result.

The rest of paper is as follows. Section 2 presents a general view of ubiquitous learning at the Mexican universities. Section 3 describes the general theory of hypothesis tests. Section 4 shows the main results obtained. Finally, Section 5 presents the conclusions and the future work.

\section{Ubiquitous Learning View in the Mexican College Context}

Delia Crovi [7] shows a report of a poll made to 382 students of the National Autonomous University of Mexico, of both sexes, between 17 and 24 years old, and of all the areas of knowledge taught in that university, on the use of cellular telephony. Among the results of the study are that $90 \%$ of the students surveyed have at least one cell phone, and that the majority of them has been using the phone for several years (from 6 to 10 years). Another result, important for the present work, is that among the 
benefits that young people find when using a cell phone, is to reach agreements to do homework. Furthermore, students say that teachers should take advantage of the cell phone potential to arrange educational activities. The study concludes by indicating that students show capacity for learning and that it is necessary to analyze the contribution to learning processes of activities such as podcasts.

In another study, the Internet Association .mx conducted a survey of 1174 young people between 25 and 29 years old with the objective of "knowing the habits, needs, motivations and obstacles of Mexican Internet users to continue preparing academically" and "to investigate perceptions and opinions that the Mexican market has about online education." [8]. In the results, on one hand it is noted that the main expectation of young people to continue their education is to have flexibility in curricula and schedules. On the other hand, the survey found that the main obstacle young people face is the lack of money to remain connected to the Internet, since the packages with unlimited service offered by the telephone companies are not within reach of their possibilities. Although the majority, $49 \%$, of the people who study at present time prefer the face-to-face modality, the online modality is in second place with $36 \%$ of the preferences. Regarding the devices used to study online, it stands out that from 2016 to 2017 the use of laptops and cell phones increased from 57\% to $62 \%$ and from $6 \%$ to $13 \%$, respectively. In contrast, the use of desktops and tablets decreased from $30 \%$ to $19 \%$ and from $7 \%$ to $6 \%$, respectively. It is foreseeable that, if this trend continues, in a few years, cell phones will displace desktop computers as a study tool. The study concludes that students consider that online education is more flexible, more demanding and allows them to better focus on the topics of study.

There is a perception that face-to-face education allows interaction with teachers and peers, requires greater investment of resources, has more clarity in the subjects of study and enjoys greater prestige with companies, while online education or elearning is gaining more and more acceptance in Mexico, and extends both in the field of formal and corporate training. It is the most successful distance learning modality and the evolution of information and communication technologies have made important contributions to the increase in the quality of pedagogical methods that, without a doubt, will set the educational standard in the not too distant future. From this evolution have emerged different learning modalities such as: blended learning (blearning), learning through games (g-learning), transformative learning (t-learning), learning in the cloud (c-learning), mobile learning (m-learning), and ubiquitous learning (u-learning), among others [9].

\section{Hypothesis Tests}

One of the main objectives of inferential statistics is to make inferences regarding unknown population parameters based on the information obtained by means of data obtained from a representative sample. These inferences can be expressed in one of two ways, as estimates of the respective parameters or as a test of hypotheses relative to their values. 
The formal procedure for a hypothesis test is very similar to the scientific method in several aspects: We propose a theory relative to the specific values of one or more population parameters, then we obtain a sample of the population and compare the observation with our theory. If the observations contradict our theory, we reject the hypothesis. Otherwise we conclude that there is no statistical evidence to contradict our theory and, therefore, it is valid or that our selected sample did not detect the difference between the real values and the values of our hypothesis with respect to the population parameters.

The elements that intervene in a hypothesis test are: i) The null hypothesis $\left(H_{0}\right)$ that represents our research hypothesis or our theory. That is, the value of the parameter that we believe is true. ii) The alternative hypothesis that represents the opposite to what we believe and that is obtained by demonstrating, using data from a representative sample as evidence, that our research hypothesis or theory is false. iii) The test statistic as an estimator, which is a function of the measurements of the sample on which the statistical decision is based. The most common test statistics are: a) the mean of the sample, b) the difference between the arithmetic means of two samples, c) the variance of a sample, d) the quotient of the variances of two samples, e) the proportion of a sample and $\mathrm{f}$ ) the correlation coefficient of a sample; iv) The rejection region, which specifies the values of the test statistic for which the null hypothesis is rejected. See Figure 1.

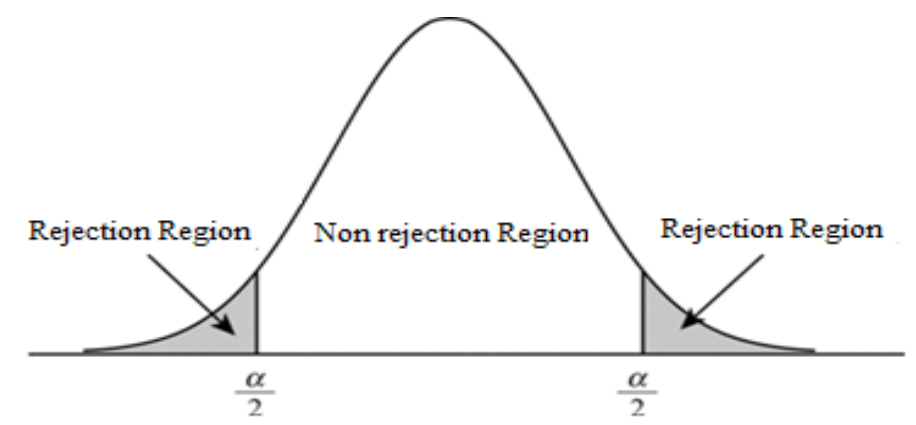

Fig. 1. Rejection and non-rejection regions of the null hypothesis

If in a particular sample, the valor calculated of the test statistic is located in the rejection region, then the null hypothesis is rejected $\left(H_{0}\right)$ and the alternative hypothesis is accepted $\left(H_{1}\right)$. Similarly, if the test statistic is not located in the rejection region, we accept $H_{0}$. The determination of an adequate rejection region for a statistical test is an interesting problem that implies major attention, as we will see in the following paragraphs.

\subsection{Common hypothesis tests}

When we want to test a hypothesis concerning to a parameter $\mu$, based in a random sample $X_{1}, X_{2}, \ldots X_{\mathrm{n}}$, the procedure for the test of hypothesis, based in the estima- 
tor $\hat{\theta}$, which has a normal or approximately normal sampling distribution with variance $\sigma_{\bar{\theta}}^{2}$, the unbiased estimators for large samples used to estimate the population mean $\mu$, and the proportion of the population $p$ that satisfy the requirements are shown in Table 1. [10]

Where in the third case, $\sigma_{1}^{2}$ and $\sigma_{2}^{2}$ are the variances of populations $1 \mathrm{y} 2$, respectively. In this case and in the last one the two samples are independent.

When the size of the population is known, the standard error must be multiplied by the finite correcting factor $\sqrt{\frac{N-n}{N-1}}$. This factor can be omited when the size of sample is less than the $5 \%$ of the population size $(n<0.05 \cdot N)$. This also is applied to the estimators for the comparison of two population means $\left(\mu_{1}-\mu_{2}\right)$, and to the comparison of two proportions of the population $\left(p_{1}-p_{2}\right)$.

Table 1. Expected values and standard errors of the more common estimators

\begin{tabular}{|c|c|c|c|c|}
\hline Target parameter $\boldsymbol{\theta}$ & Sample size(s) & Point estimator $\widehat{\boldsymbol{\theta}}$ & $\boldsymbol{E}(\widehat{\boldsymbol{\theta}})$ & Standard error $\boldsymbol{\sigma}_{\widehat{\boldsymbol{\theta}}}$ \\
\hline$\mu$ & $n$ & $\bar{X}$ & $\mu$ & $\frac{\sigma}{\sqrt{n}}$ \\
\hline$p$ & $n$ & $\hat{p}=\frac{X}{n}$ & $p$ & $\sqrt{\frac{p q}{n}}$ \\
\hline$\mu_{1}-\mu_{2}$ & $n_{1}$ and $n_{2}$ & $\bar{X}_{1}-\bar{X}_{2}$ & $\mu_{1}-\mu_{2}$ & $\sqrt{\frac{\sigma_{1}^{2}}{n_{1}}+\frac{\sigma_{2}^{2}}{n_{2}}}$ \\
\hline$p_{1}-p_{2}$ & $n_{1}$ and $n_{2}$ & $\hat{p}_{1}-\hat{p}_{2}$ & $p_{1}-p_{2}$ & $\sqrt{\frac{p_{1} q_{1}}{n_{1}}+\frac{p_{2} q_{2}}{n_{2}}}$ \\
\hline
\end{tabular}

There are different rejection regions, according to how $H_{0}$ is formulated. For example, suppose that $\mathrm{H}_{0}: \mu=\mu_{0}$ where $\mu_{0}$ is the valor that we believe the mean of the population has. The alternative hypothesis can be: $H_{1}: \mu \neq \mu_{0}$, or $H_{1}: \mu>\mu_{0}$, or even $H_{1}: \mu<\mu_{0}$. For each case, there are different rejection regions for $H_{0}$. For this case, the rejection region is shown in Figure 2a. The case for $H_{0}: \mu \neq \mu_{0}$, or $H_{0}: \mu<\mu_{0}$ or $H_{0}: \mu>\mu_{0}$ or $H_{0}: \mu \geq \mu_{0}$ or $H_{0}: \mu \leq \mu_{0}$ can be deduced in a similar way. The rejection region for the cases $H_{0}: \mu<\mu_{0}$, and $H_{0}: \mu \leq \mu_{0}$ is presented in the figure $2 \mathrm{~b}$. The rejection region for the case $H_{0}: \mu \neq \mu_{0}$ is shown in Figure 2c. The rejection region for the cases $H_{0}: \mu>\mu_{0}$ or $H_{0}: \mu \geq \mu_{0}$ is shown in Figure $2 \mathrm{~d}$.

These rejection regions are determined when the valor $\propto$ is selected, since with this valor $z_{\text {critical }}\left(\mathrm{z}_{\mathrm{c}}\right.$ in Figures $2 \mathrm{c}$ and $2 \mathrm{~d}$ ) is determined. $z_{\mathrm{c}}$ is called the critical value. If $H_{0}$ is true, the estimator $\bar{\mu}$ has a normal or approximately normal distribution, with arithmetic mean $\mu_{0}$ and variance $\sigma_{\bar{\mu}}^{2}$. For example, for the case where $H_{0}: \mu=\mu_{0}$ and $H_{1}: \mu \neq \mu_{0}$, we want that $P($ error of type $\mathrm{I})=\alpha$, then $\mathrm{z}_{\mathrm{c}}=\mu_{0} \pm \mathrm{Z}_{\alpha} \cdot \sigma_{\bar{\mu}}$ is an adequate election for $\mathrm{z}_{\mathrm{c}}$, where $\mathrm{z}_{\alpha}$ is the valor such than $\mathrm{P}\left(\mathrm{z}>\mathrm{Z}_{\alpha}\right)=\alpha$, and $-\mathrm{Z}_{\alpha}$ is the value such than $\mathrm{P}\left(\mathrm{z}>-\mathrm{Z}_{\alpha}\right)=\alpha$ when $z$ has a standard normal distribution $(\mu=0$ and $\sigma=1), \mathrm{z}$ measures the number of standard deviations between $\bar{\mu}$ and $\mu_{0}$. The three cases remaining are deduced of a similar form [10]. 


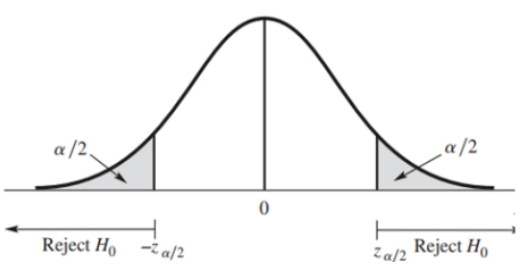

Fig. 2a

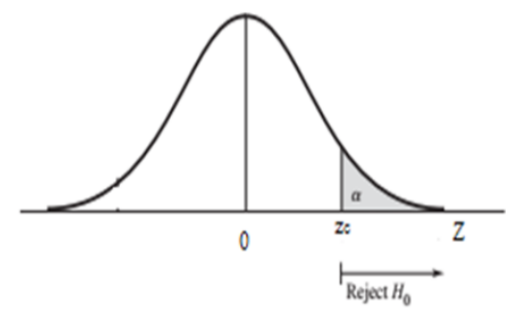

Fig. 2c

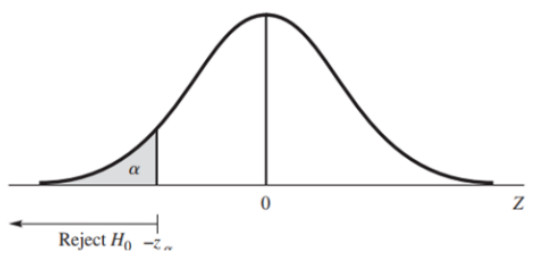

Fig. 2b

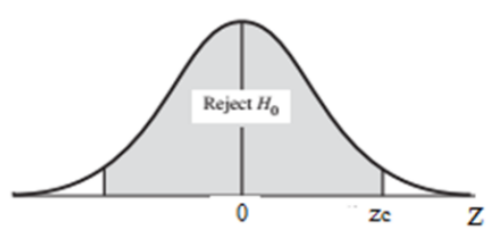

Fig. 2 d

Fig. 2. $2 \mathrm{a}, 2 \mathrm{~b}, 2 \mathrm{c}$, and $2 \mathrm{~d}$

Cases like the ones belonging to Figure $2 \mathrm{a}$ y $2 \mathrm{~d}$ are called alternative of two tails; cases like the one belonging to the Figure $2 \mathrm{~b}$ are called inferior tail alternative, and cases like the one belonging to the Figure $2 c$ are called superior tail alternative. The test statistics for these four cases is $Z_{\text {test }}=\frac{\bar{\mu}-\mu_{0}}{\sigma_{\bar{\mu}}}$, while the rejection regions for each one of the four cases are, respectively, $\mathrm{z}<-\mathrm{Z}_{\alpha}, \mathrm{z}>\mathrm{z}_{\alpha} ; \mathrm{z}>-\mathrm{Z}_{\alpha}$, and $\mathrm{z}<\mathrm{z}_{\alpha}$. When the sample size is small, and we do not know the population standard deviation $(\sigma)$, the adequate test statistic is $t_{\text {test }}=\frac{\bar{\mu}-\mu_{0}}{S_{\bar{\mu}}}$, because the $t$ distribution has a density function very much similar like the standard normal density (except that the tails are thicker) as it is shown in Figure 3 [10].

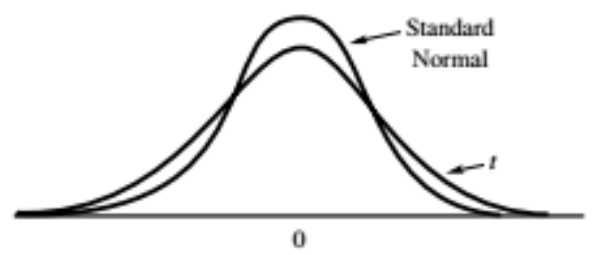

Fig. 3. A comparison of the standard normal and $t$ density functions

Finally, a type I error is committed if $\mathrm{H}_{0}$ is rejected being true. The probability of committing a type I error is indicated by $\alpha$ and is called the level of significance associated with the test. An error of type II is committed if $H_{0}$ is accepted being false. The probability of committing an error of type II is indicated by $\beta$ and can only be calcu- 
lated after having obtained a specific value for the study parameter. The complement of the probability of type II error is called the power of the statistical test.

\section{$4 \quad$ Results}

In this stage four of the "Statistics-to-Go" project we have designed four DLTs to test hypotheses of hypothetical values of parameters of a normal distribution using a sample randomly selected from a population of interest. These digital learning tools are added to those previously designed in the previous stages of the project, thus covering the descriptive and inferential statistics that are taught in the statistics courses at the University of Sonora.

\subsection{Hypothesis test for the population arithmetic mean}

With the first object, students can test hypotheses of hypothetical values of the population arithmetic mean. As stated before, students only have to fill in the statistical data required in the DLT, choose one of the four options for $H_{0}$, which were mentioned in the previous section, and press the button "Compute". Figure 4a shows the DLT screen with statistical data from a population of size $N$ unknown, a sample of size $n=30$ individuals, with arithmetic mean $\bar{X}=6.1$, and standard deviation $S=$ 0.5 . We want to test the hypothetic value of $\mu_{0}=6.0$ as a population arithmetic mean with a level of significance of $5 \%(\propto=0.05)$. Once the data is introduced in the DLT, the button "Compute" is pressed, and the information shown in Figure $4 \mathrm{~b}$ is obtained. The DLT indicates that a two-tailed test (see figure 4a) was performed, with a level of significance $\alpha=0.05$ ( 0.025 for each tail), with a test statistic of $z_{\text {test }}=1.0954$, and critical values of $z_{\text {critical }}= \pm 1.9604$. With this data, the DLT concludes that " $H_{0}$ is not rejected". This means that there is not enough statistical evidence to reject the null hypothesis. Therefore, the null hypothesis $\left(H_{0}\right)$ is accepted and the alternative hypothesis $\left(H_{1}: \mu_{0} \neq 6.0\right)$ is rejected.

Figures $4 \mathrm{c}$ and $4 \mathrm{~d}$ show a case when the size of the sample is small $(n<30)$, and both the size and the standard deviation of the population are unknown. Here it is necessary to use the $t$ distribution (see Figure 3). The data of the sample are: $n=$ $10 ; \bar{X}=86.3 ; S=2.58 ; \alpha=0.05$; and $\mu_{0}=88$. With this data, the output of the DLT concludes that it realized an one-tailed test, and that the tail is in the left side of the $t$ distribution (see figure 2b), with a level of significance $\alpha=0.05(0.05$ for the left tail), with a test statistic of $t_{\text {test }}=-2.0837$, and a critical value of $t_{\text {critical }}=$ -1.8331 . With this data, the DLT concludes that " $H_{0}$ is rejected, and $H_{1}$ is accepted". This means that there is enough statistical evidence to ensure that the null hypothesis is false, so it is recommended to accept $H_{1}$. Now, with the test statistic given by the DLT (-2.0837), students can deduce that population arithmetic mean is minor than 88 , and they could propose another hypothetic value for the arithmetic mean of the population. For example, $\mu_{0}<87.5$, then students can calculate the type II error, using $t=\frac{86.3-87.5}{\sqrt{2.58 / 10}}=-2.3625$ to obtain $\beta=0.0265$, (the probability that null hy- 
pothesis be false), and based of this error, they could make the decision of taking or not this value as the arithmetic mean of the population.

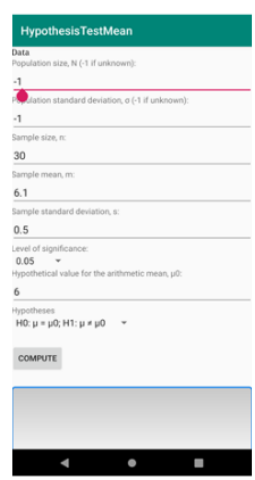

Fig. 4a

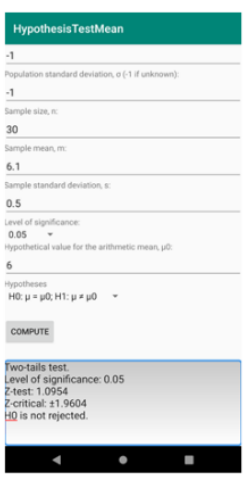

Fig. 4b

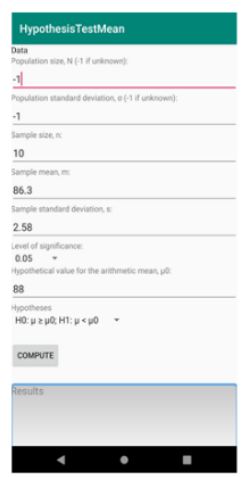

Fig. 4c

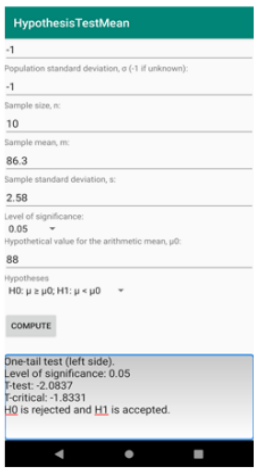

Fig. 4d

Fig. 4. $4 a, 4 b, 4 c$ and $4 d$

\subsection{Hypothesis test for the proportion of a population}

With the second DLT, students can test hypothetic values for the proportion of a population. For example, if there is a sample data of size $n=30$ individuals, with a success number of $X=10$, this implies that the sample proportion is $p=\frac{10}{30}=$ 0.33333 . Then, suppose students want to test the null hypothesis $\hat{p}=0.5$ versus the alternative hypothesis $\hat{p}<0.5$ (That is, $H_{0}: \hat{p}=0.5$, and $H_{1}: \hat{p}<0.5$ ). Figure 5a shows the screen of the DLT after the input data was entered, and Figure $5 \mathrm{~b}$ presents the output data of the DLT. In this case, the DLT indicates that it performed a one-tailed test, and the tail is in the left side of the $\mathrm{z}$ distribution (see figure $2 \mathrm{~b}$ ), with a level of significance $\alpha=0.05$ ( 0.05 for left tail), with a test statistical of $z_{\text {test }}=-1.8261$ and critical value of $z_{\text {critical }}=-1.6449$. With this data, the DLT concludes, " $H_{0}$ is rejected and $H_{1}$ is accepted". That is, there is enough statistical evidence to ensure that the null hypothesis is false. 


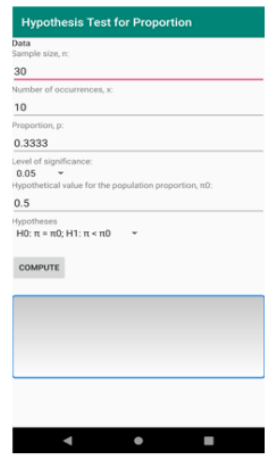

Fig. 5a

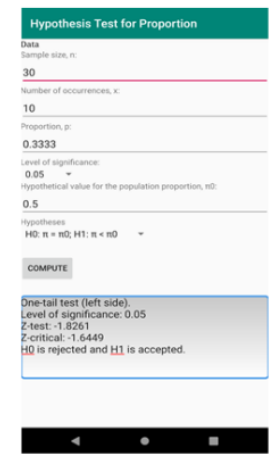

Fig. 5b

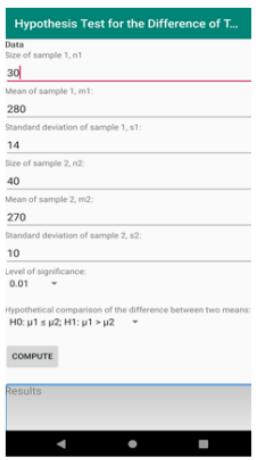

Fig. 5c

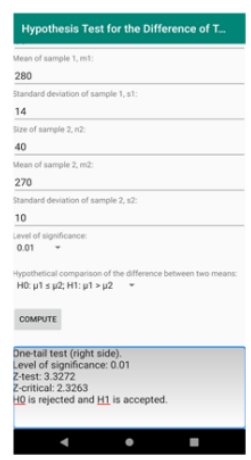

Fig. 5d

Fig. 5. $5 \mathrm{a}, 5 \mathrm{~b}, 5 \mathrm{c}, 5 \mathrm{~d}$

\subsection{Hypothesis test for the difference between two arithmetic means of the population}

With the third DLT, students can test hypothetical values for the difference between two population arithmetic means. Figure $5 \mathrm{c}$ shows the screen of the DLT with the data of two independent samples. The first one has $n_{1}=30$ individuals, $\bar{X}_{1}=280$, and $S_{1}=14$, and the second sample has $n_{2}=40$ individuals, $\bar{X}_{2}=270$, and $S_{2}=10$. With a level of significance $\alpha=0.01$. Suppose that students want to test the null hypothesis that both two samples have not been selected from two populations with equal arithmetic means. Thus, students want to prove that the arithmetic mean of the first population can be smaller than the arithmetic mean of the second population, versus the alternative hypothesis, that the arithmetic mean of the first population is bigger than the arithmetic mean of the second population. In symbols, $\mathrm{H}_{1}: \mu_{1}>\mu_{2}$ and $\mathrm{H}_{0}: \mu_{1} \leq \mu_{2}$. Figure $5 \mathrm{~d}$ shows the output data of the DLT, where it can be observes that statistical test is of one tail, and that the tail is in the right side of the normal distribution (see Figure 2a), with a level of significance $\alpha=0.01(0.01$ for right tail), statistical test of $z_{\text {test }}=3.3272$, and critical value of $z_{\text {critical }}=2.3263$. With this data, the DLT concludes: " $H_{0}$ is rejected and $H_{1}$ is accepted". That is, there is not enough statistical evidence as to accept the null hypothesis, and therefore, we must accept the alternative hypothesis.

\subsection{Hypothesis testing for the difference between two proportions of population}

With the fourth DLT, and in a similar way to the previous case, students can test if two-population proportion are not different. Figure 6a shows the screen of the DLT after the input data was entered. In this particular case, $n_{1}=50$ individuals, the success number is $X_{1}=10$, therefore sample proportion is $\hat{p}_{1}=0.2$. The second sample has $n_{2}=50$ individuals, the number of success of $X_{2}=15$, and $\hat{p}_{2}=0.3$, with a level 
of significance $\alpha=0.01$. Suppose that students want to test as the null hypothesis that the first population proportion is smaller or equal than second population proportion. That is, $\mathrm{H}_{0}: \pi_{1} \leq \pi_{2}$ versus $\mathrm{H}_{1}: \pi_{1}>\pi_{2}$. Figure $6 \mathrm{~b}$ shows the output data of the DLT, where it is noted that statistical test realized is of one tail, and that the tail is in the left side of the normal distribution (see Figure 2a), with a level of significance $\alpha=$ 0.01 ( 0.01 for left tail), statistical test of $z_{\text {test }}=-1.1547$, and critical value of $z_{\text {critical }}$ $=2.3263$. With this information, the DLT concludes: " $H_{0}$ is rejected and $H_{1}$ is accepted". With this evidence, it can be deduced that there is not enough statistical evidence for accept the null hypothesis. Therefore, the alternative hypothesis is accepted, and the null hypothesis is rejected.

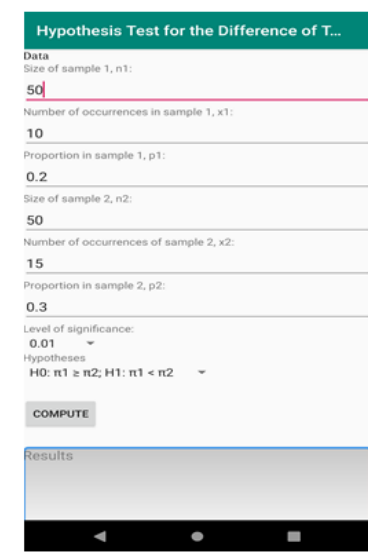

Fig. 6a

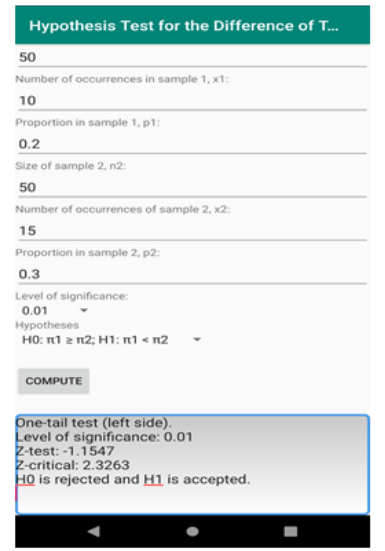

Fig. $6 b$

Fig. 6. $6 a$ and $6 b$

\section{$5 \quad$ Conclusions and Future Work}

We have designed four DLTs that will allow teachers and students to perform hypothesis tests in a simple way, without cumbersome calculations and making decision making less difficult, thus contributing to the learning of this important topic. Certainly, the efficient use of these DLTs will generate a greater interest in the subject. The students can develop the activities in an asynchronous and synchronous way, whether the teacher is present or not. We consider students have the ability and obligation to build their own knowledge and make it durable.

The design of these DLTs covers a very important issue of differential statistics related to decision making in the face of a given problem. Also, we expand the number of DLTs to cover most of the topics of descriptive and inferential statistics. 
Having these DLTs in the mobile device does not guarantee the learning of the subject, they have to be used frequently. Moreover, the student must study the topic in a good book of applied statistics recommended by the teacher.

As future work, we will design DLTs to perform analysis of variance and covariance. These are also statistical tools widely used in several areas of knowledge. The analysis of variance provides the variation of the variable of interest, in explainable sources by some factors and the variation due to sources for which the researcher has no control, cannot measure and cannot explain or attribute to any particular factor, variations that make up the so-called experimental error. The analysis of covariance allows knowing the effect of a categorical independent variable on a quantitative dependent variable (response variable), eliminating the effect that another quantitative variable has on the latter (concomitant variable).

\section{References}

[1] University of Sonora. Plan Institucional de Desarrollo 2017-2021 [Institutional Development Plan 2017-2021]. pp 25-26. 2017. Available in https://www.unison.mx/institucional/pdi2017-2021.pdf . Last view: March 05, 2019

[2] University of Sonora. Modelo Educativo 2030 [Educational Model 2030]. 2018. pp 21-22. Available

https://www.unison.mx/institucional/marconormativo/reglamentosacademicos/MODELO EDUCATIVO 2030.pdf. Last view: March 05. 2019.

[3] Zhao, X. et al. Adaptive Content Delivery in Ubiquitous Learning Environment. The 6th IEEE International Conference on Wireless, Mobile and Ubiquitous Technologies in Education (IEEE WMUTE 2010). Kaohsiung, Taiwan. https://doi.org/10.1109/wmute.2010.10

[4] Whyte, S. Digital tools for interactive teaching in languages with technology. ITILT miniguide. 2017. Available in http://www.itilt2.eu/pages/docs/Guides/2_Tools.pdf. Last view: March 05, 2019

[5] Tapia-Moreno F.J., et al. "Elaboration of statistics learning objects for mobile devices", International Journal of Interactive Mobile Technologies, 2012, Vol. 6, No. 2

[6] Tapia-Moreno F.J., Villa-Martinez H.A., "Learning Confidence Intervals with Mobile Devices", International Journal of Interactive Mobile Technologies. Volume 7, Issue 4, October 2013. https://doi.org/10.3991/ijim.v7i4.2788

[7] Delia Crovi. Jóvenes universitarios y telefonía celular [Young college students and cell phones]. Revista Mexicana de Comunicación, January 16. 2012. Available in http://mexicanadecomunicacion.com.mx/rmc/2012/01/16/jovenes-universitarios-ytelefonia-celular/. Last view: February 27, 2019

[8] Asociación de Internet .mx. La educación en línea en México 2017 [Online Education in Mexico]. Available http://mhapps01.cloudapp.net/amipci/images/EstudioEducacionenLinea2017.pdf. Last view: February 27, 2019

[9] Marcos Recio J.C., Alcolado Santos J. A New Educational Paradigm: From e-learning to Cloud Learning (c-learning). Knowledge in the Cloud. 3rd International Conference on Education and New Learning Technologies. Barcelona. Spain. 2010

[10] Mendenhall W. et al. Estadistica Matematica con Aplicaciones [Mathematical Statistics with Applications]. Grupo Editorial Iberoamericana. Segunda edición. 1994, pp. 319-321; p. 407; pp. 290-291 and pp. 424-429 
Paper-Digital Learning Tools for Mobile Devices for Accomplish Hypothesis Testing of Statistical ...

\section{$7 \quad$ Authors}

Francisco Javier Tapia-Moreno is a full-time professor in the Mathematical Department at the Universidad de Sonora in Hermosillo, Mexico. His main research interests are in e-learning, m-learning, and u-learning in statistics, and Bayesian Nets.

Hector Antonio Villa-Martinez is a full-time professor in the Computer Science program at the Universidad de Sonora in Hermosillo, Mexico. His main research interests are in Mobile Computing and Computer Graphics.

Article submitted 2019-03-09. Resubmitted 2019-04-04. Final acceptance 2019-04-04. Final version published as submitted by the authors 